\title{
Dosimetric evaluation of field-in-field and sliding- window IMRT in endometrium cancer patients with a new approach for the conformity index
}

\author{
A. Özseven* and E. Elif Özkan \\ Suleyman Demirel University Research and Application Hospital Department of Radiation Oncology, Isparta, \\ Turkey
}

\begin{abstract}
Background: Purpose of the study is to evaluate field-in-field (FIF) and slidingwindow intensity modulated radiation therapy (SW-IMRT) treatment plans in terms of homogeneity index $(\mathrm{HI})$, conformity index $(\mathrm{Cl})$ and normal tissue doses for organs at risk (OAR) in patients with endometrial carcinoma along with deriving a new expression for $\mathrm{Cl}$. Materials and Methods: Four different FIF plans with different MLC (multi-leaf collimator) margins and SW-IMRT plan were compared in 20 endometrial cancer patients who underwent adjuvant pelvic radiotherapy with $50.4 \mathrm{~Gy}$ in 28 fractions. Normal tissue doses were assessed for OAR from dose volume histograms (DVHs). $\mathrm{HI}$ and $\mathrm{Cl}$ values were calculated according to the reports of International Commission on Radiation Units and Measurements (ICRU)-83, Radiation Therapy Oncology Group (RTOG) 1993 and ICRU-62. A new $\mathrm{Cl}$ was developed and named CIOPT standing for optimized conformity index. Results: SW-IMRT provided a significantly better $\mathrm{Cl}$ in comparison to other FIF plans $(p<0.001)$. Conventional-FIF (C-FIF) was the most homogeneous plan compared to all other FIF plans and SW-IMRT $(p \leq 0.001)$. The absolute volume of small intestine that received $\geq 45 \mathrm{~Gy}(\mathrm{~V} 45)$ and doses received by a $30 \%$ volume of rectum (D30) were dramatically reduced in SW-IMRT $(p<0.001)$. Conclusion: MLC margins had a substantial influence on OAR doses, $\mathrm{HI}$ and $\mathrm{Cl}$. A close proximity of $\mathrm{Cl}_{\text {OPT }}$ to 1 indicated that this formulation of $\mathrm{Cl}$ was a useful plan evaluation tool, which was also compatible with the RTOG 1993 and ICRU-62 reports.
\end{abstract}

- Original article

${ }^{*}$ Corresponding authors:

Alper Özseven, Ph.D.,

E-mail:

alperozseven@sdu.edu.tr

Revised: March 2020

Accepted: June 2020

Int. J. Radiat. Res., October 2020; 18(4): $853-862$

DOI: 10.18869 /acadpub.ijrr.18.4.853

Keywords: Conformity index, endometrium cancer, field-in-field, homogeneity index, IMRT.

\section{INTRODUCTION}

Endometrial cancer is the fourth most common gynaecological cancer worldwide (1). Surgery and adjuvant radiotherapy in cases at intermediate or high risk for loco-regional recurrence is accepted as the standard of care based on sufficient outcomes (1-3).Two large randomised controlled trials defined the subgroups that benefit from adjuvant therapy, such as (1) poorly differentiated tumours (2), presence of lymphovascular space invasion, and (3) outer third myometrial invasion; where the patients were aged $\geq 50$ years with any two risk factors, or aged $\geq 70$ with any one risk factor $(1,2)$.

Large target volumes in patients, including the intestine, bladder and rectum, result in gastrointestinal and urinary complications (4). Intensity modulated radiation therapy (IMRT) is the preferred technique, providing adequately matching dose distributions for irregularly shaped target volumes and sufficient protection of the organ at risk (OAR), such as in gynaecologic malignancy radiation treatments (5). Chronic complications have been reported to decrease significantly with IMRT, without any 
cost in terms of disease control and survival $(6,7)$. Identifying and contouring the target volume and OAR sensitivity is essential for IMRT planning. Target volume and OAR were defined by the International Commission on Radiation Units and Measurements (ICRU)-83 report (8). A consensus-based guideline for clinical target volume (CTV) in adjuvant radiotherapy of endometrial carcinoma was suggested in early 2000 's (9). Three levels for prescribing and reporting were recommended by ICRU-83. Level -I is for two-dimensional (2D) planning. Level-II involves 3D imaging for planning, dose calculation with inhomogeneity corrections and evaluation of dose volume histograms (DVHs). Level-III includes tumour control probability and normal tissue (NT) complication probability (NTCP) (8). 3D-conformal radiotherapy (3D-CRT) with the four-field box technique and IMRT are the most commonly used methods, each of which has its own advantages and disadvantages (10). With the four-field box technique, the bladder is exposed to the majority of the prescribed dose, and frequently a considerable part of the rectum is in the treatment field. By contrast, IMRT is a well-developed technique where critical organs just around the planning target volume (PTV) can be very well protected despite an irregular shaped PTV (11). Field-infield (FIF) radiotherapy operates in the reverse way to inverse IMRT and is accepted as static IMRT. By applying an appropriate number of segments on 3D-CRT, a FIF plan can be obtained, and the negative effects of CRT in terms of conformity and homogeneity can be eliminated. Attempts have been made to determine which technique is superior with regard to target coverage, homogeneity, conformity and normal tissue dose $(7,10,11)$.

Normal tissue dose assessments are usually made by using the suggestions of the Radiation Therapy Oncology Group (RTOG) and Quantitative Analyses of Normal Tissue Effects in the Clinic (QUANTEC). A quantitative assessment should be made to evaluate the homogeneity and conformity of the treatment plan. Homogeneity index (HI) and conformity index (CI) terms have been the most objective scoring tools that studies have utilised to measure the overall treatment plan. The main aim of radiotherapy treatment is to ensure an optimum dose distribution, giving maximal tumour coverage with homogeneous dose distribution and minimum dose to adjacent normal tissue (12,13). The concept of HI was proposed by RTOG in 1993, with the maximum isodose in target and the Reference Isodose (RI) chosen by the clinician (14). Some derivatives have been proposed in subsequent years, with the most illustrative and useful one being in the ICRU-83 report, which was accepted as the most common formula in the literature (8).

The other useful scoring tool for assessing the treatment plan is CI. The term was first proposed by Shaw etal., who cooperated with the RTOG in 1993, and it was defined with same terminology in Report 62 by the ICRU $(14,15)$. Both RTOG and ICRU-62 reports suggested a similar formula, which provides the same CI result. Feuvret et al. reviewed the concept by analysing the proposed approaches in detail (16). Subsequently, Knoos et al. submitted a new derivative which was the inverse of the RTOG suggestion (17). With the development of techniques in radiation delivery, studies continued to offer some new formulations (12). Lefkopoulos et al. as the Saint-Anne-Lariboisière -Tenon (SALT) group, introduced an expression which mainly evaluated the relation of RI volume with target volume (18). Lomax and Scheib proposed a CI which took the irradiation of healthy tissue into consideration by calculating the reference isodose volume outside the target volume (19). It is widely accepted that all the aforementioned CIs had some limitations in evaluating the coverage of target volume, and the irradiation of healthy tissues. On the other hand, van't Riet et al. defined CI, as conformation number (CN), which assessed both target volume and critical structures adjacent to the target (20).

This study evaluated FIF and sliding-window IMRT (SW-IMRT) treatment plans in terms of HI, $\mathrm{CI}$ and normal tissue doses for OAR in endometrial carcinoma patients, along with deriving a new optimised CI (CIOpT). In addition, the effect of MLC margins on normal tissue dose, $\mathrm{HI}$ and CI were investigated in FIF plans.

Int. J. Radiat. Res., Vol. 18 No. 4, October 2020 


\section{MATERIALS AND METHODS}

Radiotherapy treatment planning data of 20 endometrial cancer patients, treated with adjuvant pelvic radiotherapy in the clinic, were retrospectively assessed. For each patient, four different FIF plans and a SW - IMRT plan were created. These five plans were compared in terms of normal tissue dose constraints, $\mathrm{CI}$ and HI. In addition, a new expression "CIOPT" standing for Optimized Conformity Index was derived which took into consideration the volume of the specified reference isodose, its intersections with the PTV and normal tissue, and its missing volume in the PTV. The normal tissue dose was evaluated using the RTOG and QUANTEC suggestions. HI calculations were completed by using descriptions in RTOG 1993 and ICRU-83. In addition, CI values were calculated with the existing RTOG 1993 and ICRU-62 recommendations and the new proposed formulation denominated as $\mathrm{CI}_{\mathrm{OPT}}$

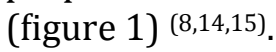

\begin{tabular}{|c|c|}
\hline $\mathrm{HI}_{\mathrm{RTOG}}=\frac{\mathrm{I}_{\max }}{\mathrm{RI}}$ & $\begin{array}{l}I_{\max }=\text { maximum isodose in the target } \\
\mathbf{R I}=\text { reference isodose }\end{array}$ \\
\hline $\mathrm{HI}_{\mathrm{ICRU}}=\frac{(\mathrm{D} 2-\mathrm{D} 98)}{\mathrm{Dp}}$ & $\begin{array}{l}D_{2}=\text { minimum dose to } 2 \% \text { of the target } \\
\text { volume indicating the "near-maximum dose" } \\
\text { D98 }=\text { minimum dose to } 98 \% \text { of the target } \\
\text { volume indicating the "near-minimum dose" } \\
D_{\mathbf{p}}=\text { prescribed dose }\end{array}$ \\
\hline $\mathrm{CI}_{\mathrm{RTOG}}=\frac{\mathrm{V}_{\mathrm{RI}}}{\mathrm{TV}}$ & $\begin{array}{l}V_{R I}=\text { volume of reference isodose } \\
\mathrm{TV}=\text { target volume }\end{array}$ \\
\hline $\mathrm{CI}_{\text {ICRU }}=\frac{\mathrm{TV}}{\mathrm{V}_{\mathrm{PTV}}}$ & $\begin{array}{l}\text { TV = treated volume (volume enclosed } \\
\text { by the isodose surface) } \\
V_{\text {PTV }}=\text { volume of planning target volume }\end{array}$ \\
\hline $\mathrm{CI}_{\mathrm{OPT}}=\frac{\mathrm{V}_{\text {OVERLAP }}-\mathrm{V}_{\text {MISSING }}}{\mathrm{V}_{\text {PTV }}}-\frac{\mathrm{V}_{\text {EXCESSIVE }}}{\mathrm{V}_{\mathrm{RI}}}$ & 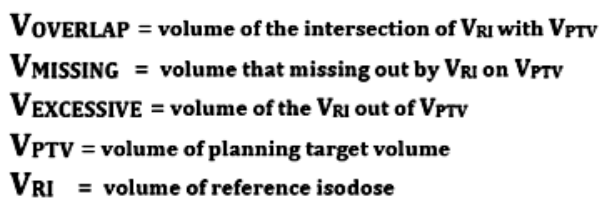 \\
\hline
\end{tabular}

Figure 1. Comparison of homogeneity index and conformity index expressions.

\section{Patient simulation and contouring}

All but three patients were scanned in a prone position. Arms were located on the chest for patients, scanned in a supine position. Computerised tomography (CT) images were obtained with a $2.5-\mathrm{mm}$ slice thickness from the upper abdomen to the bottom of the perineum with a full bladder using CT scanner (General Electric Medical Systems). Intravenous contrast was not used.

The preoperative tumour bed, vagina, paravaginal soft tissue through the lower end of the obturator fossa, as a lower limit, was delineated as the CTV for the tumour bed $\left(\mathrm{CTV}_{\mathrm{TM}}\right)$. Low risk nodal CTV $\left(\mathrm{CTV}_{\mathrm{N}}\right)$ included common, external, and internal iliac nodal groups, with an upper limit at the bottom of vertebra L5 to the presacral lymph node group 
through the S3 vertebra as a lower limit. The PTV was generated by adding a $7 \mathrm{~mm}$ margin to $\left(\mathrm{CTV}_{\mathrm{TM}}\right)$ and $\left(\mathrm{CTV}_{\mathrm{N}}\right)$ in all directions. Bladder, rectum, bilateral femoral heads and the small intestine were the normal tissues contoured as OAR. All the tissue other than the PTV included in the treatment field was defined as NT. The whole peritoneal potential space of the intestine was contoured in evaluating small intestine dose parameters.

The study was approved by the Scientific Research Ethics Committee of the Medical Faculty of Suleyman Demirel University (protocol code: 11/10.01.2018). All procedures were performed in accordance with the ethical standards of the institutional research committee in alliance with the 1975 Helsinki declaration and its later amendments. The need for informed consent was waived owing to the retrospective nature of the study.

\section{Treatment planning techniques}

Treatment plans were created using the Eclipse treatment planning system on Varian DHX linear accelerator, with dose calculation by an anisotropic analytical algorithm (AAA). A total of 50.4 Gy was planned in 28 fractions as the prescribed dose. For FIF planning, $18 \mathrm{MV}$ X-rays was used with the 4 field - box classical treatment techniques. SW-IMRT treatment plans for patients in the prone position were performed with seven fields $(30,60,105,180$, $255,300-$ and 330-degree angles) by $6 \mathrm{MV}$ $\mathrm{X}$-rays. For the SW-IMRT plans for supine positioned patients, the conjugate angles of these angles were used, which were $0,75,120$, 150, 210, 240, and 285 degrees. In all 20 patients, the same optimization parameters were used in the SW-IMRT planning process. For FIF planning, four different plans were studied for each patient with four different MLC margins (the distance between the PTV edge and the MLC end in beam eye view). The MLC margins were used both in main the fields and for field-in -field segments. These four different plans were named according to the MLC margins in centimetres $(\mathrm{cm})$. The FIF plan, with the MLC margin determined by the border of anatomical structures, in the conventional radiotherapy treatment plan, was termed C-FIF, having a MLC margin more than $1.1 \mathrm{~cm}$. The FIF plans with 0.9 , 0.7 and $0.5 \mathrm{~cm}$ MLC margins were named 0.9-FIF, 0.7-FIF and 0.5-FIF, respectively. For both the FIF and SW-IMRT plans, the mean dose received by the target volume was equalised.

\section{Treatment plan analysis}

Normal tissue doses, $\mathrm{HI}$ and $\mathrm{CI}$ were evaluated from the obtained DVHs. RTOG 0418 and RTOG 1203 protocols and QUANTEC suggestions were taken into consideration in terms of normal tissue dose limits (table 1) (21-24). In both RTOG reports, the suggested limits were only for the IMRT technique. Especially in RTOG 1203 , no exact recommendations were found for the standard arm. Treatment planning of FIF combined both the standard technique and forward IMRT; therefore, these limits were taken into consideration for OARs in these treatment plans. In current study, $97 \%$ was chosen as the reference isodose for endometrial cancer treatment, a level which was also suggested by the RTOG 0418 and RTOG 1203 protocols.

\section{Derivation of CIopT}

$\mathrm{CI}_{\mathrm{OPT}}$, a new optimised formulation of $\mathrm{CI}$ was developed to represent the conformity more precisely (equation 1).

CIopt $=\frac{\text { VoVERLAP }}{V_{\text {PTV }}}-\frac{V_{\text {MISSING }}}{V_{\text {PTV }}}-\frac{V_{\text {EXCESSIVE }}}{V_{\text {RI }}}$

where,

$\mathbf{V}_{\text {PTV }}$ is the volume of planning target volume,

$\mathbf{V}_{\mathbf{R I}}$ is the volume of the specified reference isodose,

VoverLap is the volume that defines the intersection of $V_{R I}$ with $V_{\text {PTV }}$,

$\mathbf{V}_{\text {MISSING }}$ is the volume missed by $V_{\text {RI }}$ on $V_{\text {PTV }}$, and $\mathbf{V}_{\text {EXCESSIVE }}$ is the volume of $V_{\text {RI }}$ out of $V_{\text {PTV }}$, in other words, the volume of $V_{R I}$ in normal tissue.

Representation of all the volumes used in calculating $\mathrm{CI}_{\mathrm{OPT}}$, obtained after the treatment planning process for FIF and SW-IMRT plans are shown in figure 2 and figure 3, respectively. In addition, a schematic visualization of the volumes used for calculating $\mathrm{CI}_{\mathrm{OPT}}$ is shown in table 2 .

Int. J. Radiat. Res., Vol. 18 No. 4, October 2020 
It was clear from Eqn.1 that [ $\left.\mathrm{V}_{\text {OVERLAP }} / \mathrm{V}_{\mathrm{PTV}}\right]$ formed the positive part of $\mathrm{CI}_{\mathrm{OPT}}$; while $\left[\mathrm{V}_{\text {MISSING }} / \mathrm{V}_{\mathrm{PTV}}\right]$ and $\left[\mathrm{V}_{\text {EXCESSIVE }} / \mathrm{V}_{\mathrm{RI}}\right]$ were the negative part. Actually, the first two terms of equation. 1 could be rewritten with a common denominator, producing the equation in a simpler form (equitation 2).

$$
\text { CIopt }=\frac{\text { VoverLAP }-V_{\text {MISSING }}}{V_{\text {PTV }}}-\frac{V_{\text {EXCESSIVE }}}{V_{\text {RI }}}
$$

For $\mathrm{CI}_{\mathrm{OPT}}$, the more the result is close to 1 , the more the plan is highly conformal. $\mathrm{CI}_{\mathrm{OPT}}$ not only evaluates the coverage of the reference isodose on the PTV but also assesses the amount of unnecessary irradiation on healthy tissues and any cold spots on the PTV.

In three - dimensional space, the spatial distribution of the reference isodose volume could be critical. To be more precise, the location of the covered volume of the reference isodose in the Cartesian coordinate system significantly affected the conformity of the treatment plan.

Considering current treatment delivery techniques, it was accepted that the volume of PTV and volume of RI were roughly comparable in $\mathrm{cm}^{3}$. The possible scenarios where $\mathrm{CI}_{\text {OPT }}$ might generate different results are shown in detail (table 2).

\section{Statistical analysis}

Statistical analyses were performed using the SPSS software version 22 (Armonk, NY: IBM Corp., USA). From repeated measures, analysis of variance was used to find the difference between the mean values of the parameters with a normal distribution. For non-normal distributions, significance of the difference was analysed using the Friedman test and the Wilcoxon signed-rank test. A p-value $\leq 0.05$ was considered to indicate statistical significance.

Table 1. Suggested dose limits by RTOG and QUANTEC for assessing normal tissue doses.

\begin{tabular}{|c|c|c|}
\hline Normal Tissue & Dose Limits & Suggested by \\
\hline Bladder & D35 $\leq 45 \mathrm{~Gy}$ & RTOG 0418 / RTOG 1203 \\
\hline Rectum & D60 $\leq 30 \mathrm{~Gy}$ & RTOG 0418 \\
\hline Femoral Heads & D15 $\leq 30 \mathrm{~Gy}$ & RTOG 0418 \\
\hline \multirow{2}{*}{ Small Intestine } & D30 $\leq 40 \mathrm{~Gy}$ & RTOG 0418 / RTOG 1203 \\
& QUANTEC $<195 \mathrm{cc}$ & QUA \\
\hline $\begin{array}{l}\text { D35, doses received by a 35\% volume of bladder; D60, doses received by a 60\% volume of rectum; D15, doses received by a 15\% volume of femoral } \\
\text { heads; D30, doses received by a 30\% volume of small intestine; V45, the absolute volume of small intestine that received } \geq 45 \text { Gy. }\end{array}$
\end{tabular}

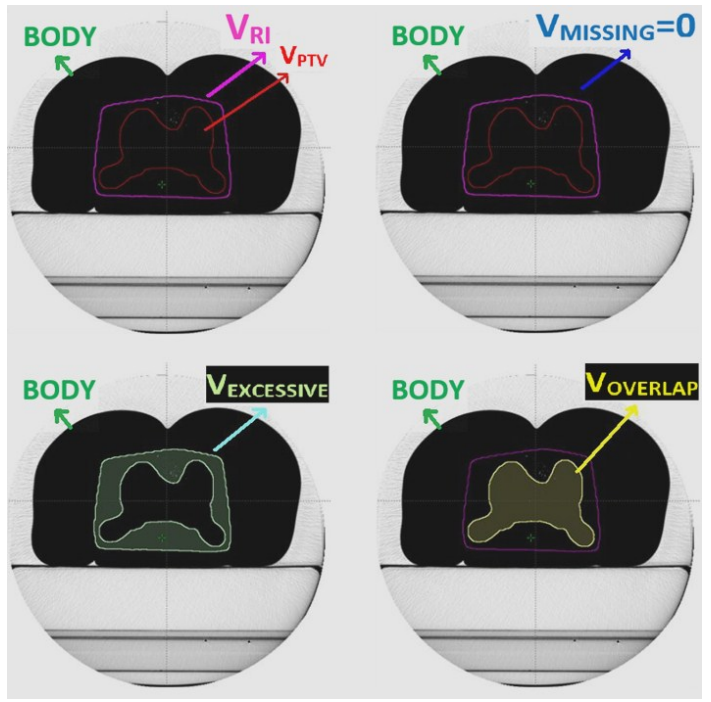

Figure 2. Axial view of the resultant volumes of $V_{R I}, V_{P T V}$, $V_{\text {OVERLAP, }} V_{\text {MISSING }}$ and $V_{\text {EXCESSIVE }}$ in the field-in-field treatment planning procedure.

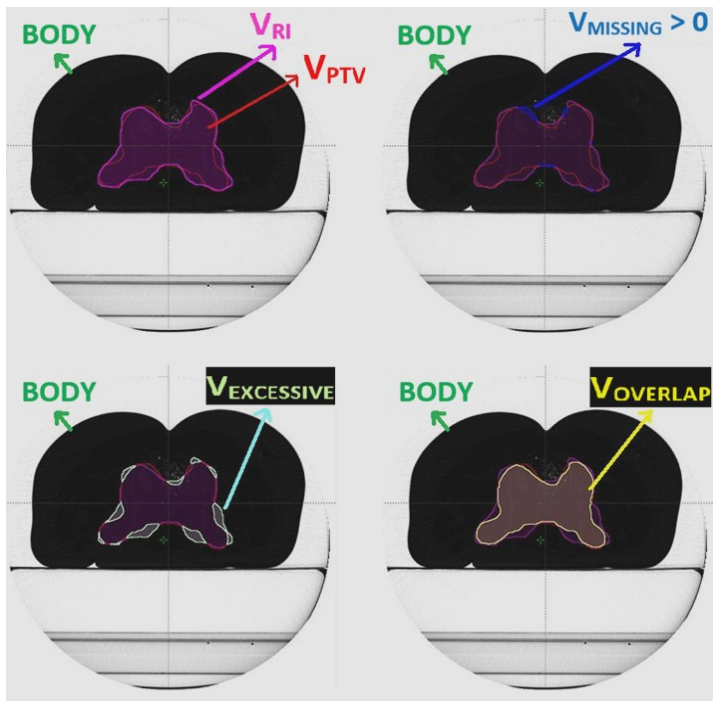

Figure 3. Axial view of the resultant volumes of $V_{R 1}, V_{P T V}$, $V_{\text {OVERLAP, }} V_{\text {MISSING }}$ and $V_{\text {EXCESSIVE }}$ in the SW-IMRT treatment planning procedure. 
Table 2. Possible scenarios for a treatment plan and $\mathrm{Cl}_{\mathrm{OPT}}$.

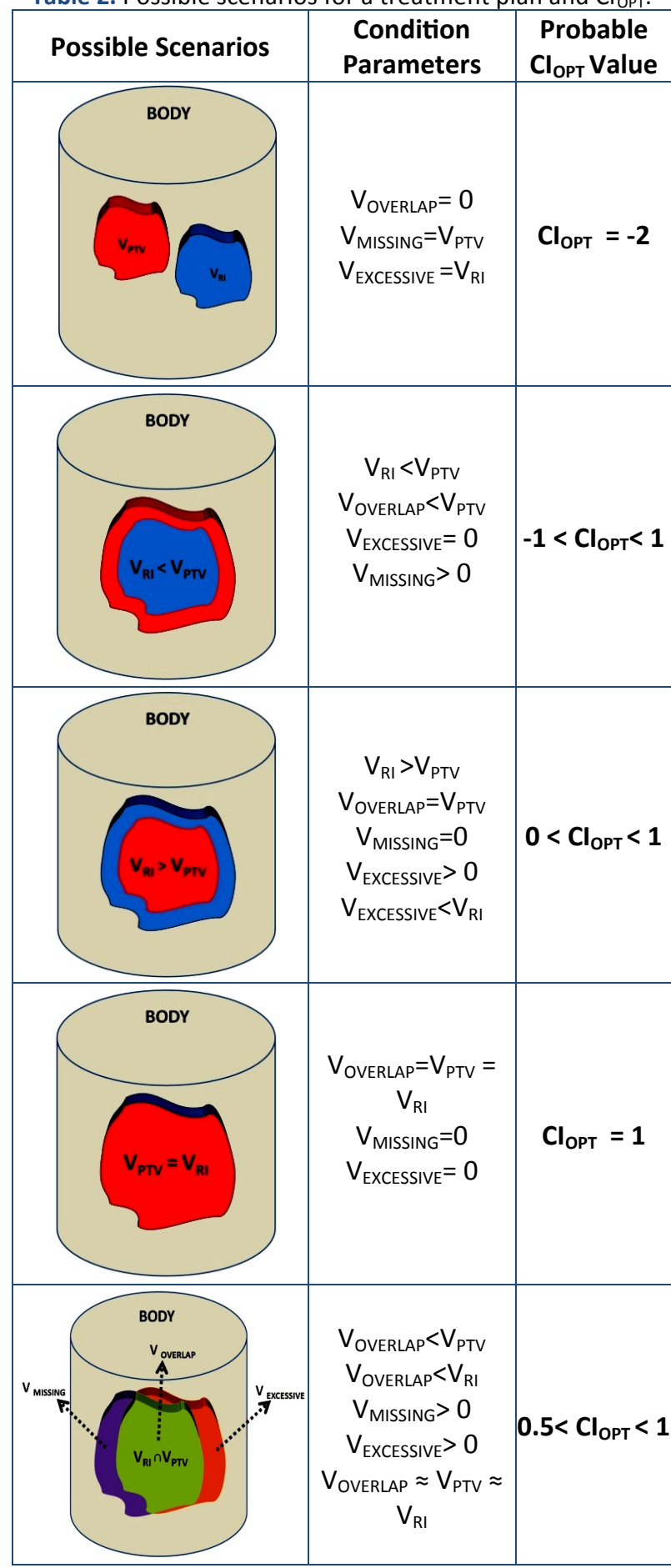

\section{RESULTS}

\section{Homogeneity and conformity indices}

The calculated mean/median $\mathrm{HI}$ and $\mathrm{CI}$ values are shown in table 3 . The C-FIF plan was the most homogeneous treatment plan with ICRU expression $(\mathrm{p}<0.001)$. The results of evaluation according to RTOG revealed a statistically significant difference in favour of all four different FIF plans, when compared with SW- IMRT in terms of homogeneity $(\mathrm{p}<0.001)$.

On the other hand, the expressions for $\mathrm{CI}$, as defined by ICRU-62 and RTOG 1993 reports, generated the same results (14,15). All the CI results showed that SW-IMRT was considerably closer to 1 , indicating better conformity $(\mathrm{p}<0.001)$.

\section{Normal tissue doses}

The mean/median values of the dose parameters that were evaluated for the comparison of OARs between the five treatment plans are summarised in table 4.

As an illustration, the superiority of SW-IMRT over FIF in terms of OARs could be seen from the DVH, in a comparison of the SW-IMRT and 0.9-FIF plans (figure 4).

\section{Bladder}

The mean results of the doses received by a $35 \%$ volume of the bladder (D35) were similar in all FIF plans $(p=0.11-0.79)$. However SW-IMRT showed a significant advantage compared to the other four FIF plans ( $\mathrm{p}<$ $0.001)$.

\section{Rectum}

The doses received by a $60 \%$ volume of rectum (D60) values were reduced considerably in SW-IMRT compared with all other FIF plans $(p<0.001)$. Although, a statistically significant difference was found between C-FIF and the other FIF plans $(\mathrm{p}<0.05)$, no significant difference was shown between 0.9-FIF, 0.7-FIF and 0.5 -FIF ( $p=0.39-0.47)$.

\section{Femoral heads}

No noticeable differences were found between the calculated mean/median doses received by a $15 \%$ volume of the femoral heads (D15) for the SW-IMRT, 0.7-FIF and 0.5-FIF plans $(\mathrm{p}=0.351$ and $\mathrm{p}=0.794$, respectively). The SW-IMRT plan was significantly superior

Int. J. Radiat. Res., Vol. 18 No. 4, October 2020 
compared to the C-FIF and 0.9-FIF plans $(\mathrm{p}<0.001$ and $\mathrm{p}=0.019$, respectively). When the D15 values of the FIF plans were compared among each other, all the resultant p-values were found to be $<0.001$, which indicated that the MLC margins had an excessive effect on femoral head doses.

\section{Small intestine}

Doses received by a $30 \%$ volume of small intestine (D30) indicated that, SW-IMRT was better than all the FIF plans for reducing the small intestine dose, except for 0.5-FIF. This difference was significant for the C-FIF and
0.9-FIF plans $(\mathrm{p}<0.002$ and $\mathrm{p}=0.013$, respectively). However, the results for the 0.7-FIF and 0.5-FIF plans were not significant ( $\mathrm{p}=0.184$ and $\mathrm{p}=0.800$, respectively). In addition, the absolute volume of small intestine that received 45 Gy and more (V45) was decreased dramatically in the SW-IMRT technique compared with the other four FIF plans, as expected $(\mathrm{p}<0.001)$. As for the femoral heads, when D30 and V45 values of the FIF plans were compared to each other, all had p-values $<0.001$, which showed that the MLC margins had a significant effect on small intestine doses.

Table 3. Comparison of five planning techniques in terms of homogeneity index (HI) and conformity index $(\mathrm{Cl})$.

\begin{tabular}{|c|c|c|c|c|c|c|}
\hline & SW-IMRT & $C-F I F$ & $0.9-F I F$ & 0.7-FIF & $0.5-F I F$ & $p$ \\
\hline$H I_{I C R U}(C)$ & $0.059 \pm 0.009$ & $0.044 \pm 0.010$ & $0.051 \pm 0.010$ & $0.060 \pm 0.010$ & $0.076 \pm 0.009$ & $<0.001^{*}$ \\
\hline$H I_{R T O G} \check{S}$ & $1.120 \pm 0.013$ & $1.082 \pm 0.009$ & $1.083 \pm 0.008$ & $1.084 \pm 0.007$ & $1.087 \pm 0.007$ & $<0.001 *$ \\
\hline$C l_{\text {ICRU/RTOG }} \not \mathbf{x}$ & $1.221 \pm 0.047$ & $2.498(2.037-3.889)$ & $2.019 \pm 0.190$ & $1.844 \pm 0.169$ & $1.675 \pm 0.149$ & $<0.001^{*}$ \\
\hline $\mathrm{Cl}_{O P T}{ }^{\circledR}$ & $0.860 \pm 0.022$ & $0.395 \pm 0.066$ & $0.489(0.318-0.606)$ & $0.521 \pm 0.063$ & $0.542 \pm 0.065$ & $<0.001^{*}$ \\
\hline
\end{tabular}

Data are presented as mean \pm standard deviation or median (minimum-maximum), whichever is applicable. The best results are represented in bold. SW-IMRT, sliding-window intensity modulated radiation therapy; $C$-FIF, field-in field plan with multi-leaf collimator (MLC) margins $>1.1 \mathrm{~cm} ; 0.9$ -FIF, field-in-field plan with $0.9 \mathrm{~cm}$ MLC margins; $0.7-F I F$, field-in-field plan with $0.7 \mathrm{~cm}$ MLC margins; $0.5-F I F$, field-in-field plan with $0.5 \mathrm{~cm}$ MLC margins, (C), homogeneity index (HI) values using the ICRU 83 report; $\breve{S}$, $\mathrm{HI}$ values using the RTOG 1993 report; $\not 2$, conformity index (CI) values using the ICRU 62/ RTOG 1993 report; ${ }^{\circledR}, \mathrm{Cl}$ values using new approach; ${ }^{*}$, statistically significant $p$-values.

Table 4. The mean/median values of the dose parameters of organs at risk (OAR) for the five treatment plans.

\begin{tabular}{|c|c|c|c|c|c|}
\hline Parameter & SW-IMRT & C-FIF & 0.9-FIF & 0.7-FIF & O.5-FIF \\
\hline Bladder D35(Gy) & $\mathbf{5 1 . 2 0 ( 4 4 . 6 7 - 5 2 . 2 0 )}$ & $51.80 \pm 0.34$ & $51.74 \pm 0.37$ & $51.81 \pm 0.38$ & $51.91 \pm 0.42$ \\
\hline Rectum D60(Gy) & $\mathbf{4 0 . 4 8} \pm \mathbf{4 . 9 9}$ & $51.50 \pm 0.38$ & $51.12(48.57-52.21)$ & $51.10(46.98-52.22)$ & $51.12(44.15-52.27)$ \\
\hline $\begin{array}{c}\text { Small Intestine } \\
\text { D30(Gy) V45(cc) }\end{array}$ & $\mathbf{2 9 . 9 1 \pm 7 . 0 8}$ & $31.57(15.60-51.98)$ & $33.99 \pm 12.00$ & $31.83 \pm 11.69$ & $\mathbf{2 9 . 5 7} \pm \mathbf{1 1 . 4 0}$ \\
\hline $\begin{array}{c}\text { Femoral Heads } \\
\text { D15(Gy) }\end{array}$ & $34.21 \pm 3.55$ & $45.69(30.81-52.20)$ & $33.81(30.13-51.28)$ & $32.29(29.58-50.80)$ & $\mathbf{3 1 . 4 9}(\mathbf{2 9} .10-49.41)$ \\
\hline
\end{tabular}

Data are presented as mean \pm standard deviation or median (minimum-maximum), whichever is applicable. The lowest results are represented in bold SW-IMRT, sliding-window intensity modulated radiation therapy; C-FIF, field-in field plan with multi-leaf collimator (MLC) margins $>1.1 \mathrm{~cm}$; 0.9 FIF, field-in-field plan with $0.9 \mathrm{~cm} \mathrm{MLC}$ margins; $0.7-F I F$, field-in-field plan with $0.7 \mathrm{~cm} \mathrm{MLC} \mathrm{margins;} 0.5-F I F$, field-in-field plan with $0.5 \mathrm{~cm}$ MLC margins; D35, doses received by a $35 \%$ volume of bladder; D60, doses received by a $60 \%$ volume of rectum; D30, doses received by a $30 \%$ volume of small intestine; V45, the absolute volume of small intestine that received $\geq 45 \mathrm{~Gy}$; D15, doses received by a $15 \%$ volume of femoral heads.

Figure 4. Comparison of dose volume histograms (DVHs) between SW-IMRT and 0.9-FIF plans.

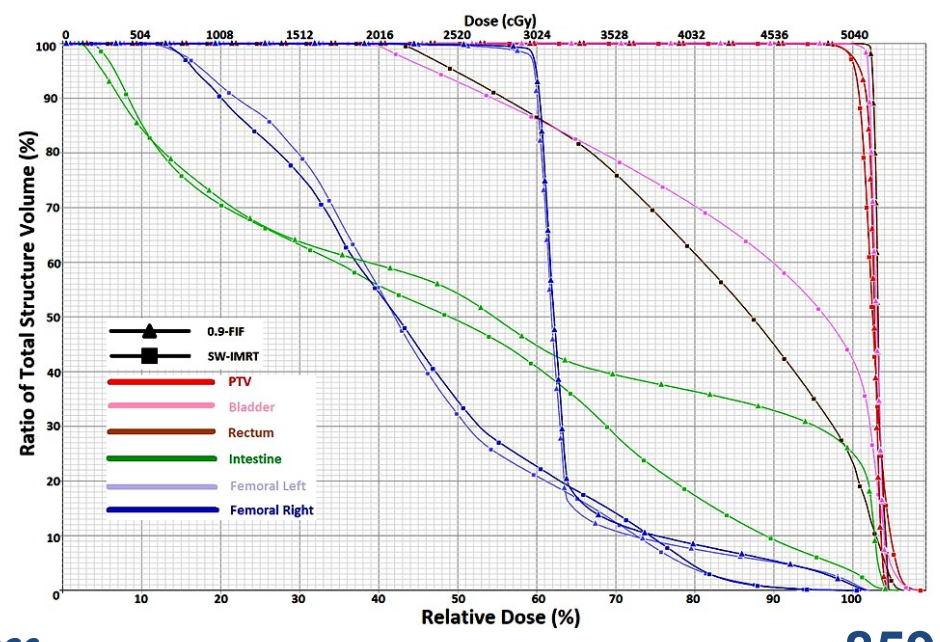




\section{DISCUSSION}

The main objective of a radiotherapy plan is the best tumour coverage, while affording healthy tissue protection. Rectum, small intestine, bladder and bone marrow toxicities need to be avoided during pelvic radiation therapy. Technical developments in radiation oncology have provided longer survival times for patients with gynaecological tumours. Long- term post-treatment life has drawn attention to any changes in intestine and bladder function that impair quality of life. This issue warrants advanced techniques, risk-adapted planning and overall plan evaluation. Optimum plans corresponding to the above requirements need an evaluation of different techniques, different angles, and even different energies for each patient (25). CI defines an easy but patient-specific formula, which compares the different plans, and adds accuracy and sensitivity to slice by slice isodose assessment and/or DVH assessment for both target volume evaluation and OAR doses.

In clinical routines, whole pelvis radiation therapy for endometrial cancer patients after surgery is delivered using FIF plans, consisting of multiple sub-segments, with doses received by the entire OAR being evaluated by analysing DVHs.

Yavas etal. compared the dosimetric data from their FIF and 3D-CRT plans in patients with early stage endometrial cancer. The FIF technique was found to be superior to 3D-CRT in terms of dose homogeneity and the doses received by OAR. In addition, a decrease in hot spots was detected with the FIF technique in the same study. IMRT has been advocated by some groups with the expectation of improving the therapeutic ratio by more precise conformity and steep dose gradients (25). Similarly, in this current study, better dose homogeneity was achieved with the FIF technique than SW-IMRT $(p<0.001)$. On the other hand, from DVH analysis, SW-IMRT results were roughly comparable to the 0.7-FIF and 0.5-FIF plans in terms of D15 dose of femoral heads ( $\mathrm{p}=0.35$ and $\mathrm{p}=0.79$, respectively) and D30 parameter of the small intestine $(\mathrm{p}=0.184$ and $\mathrm{p}=0.800$, respectively), which indicated predictably that MLC margins had a significant effect on the small intestine and femoral heads.

Heron et al. compared the four-field box 3D-CRT with 7-field IMRT. Dose exposures for the bladder, rectum and small intestine in DVH were reduced with 7-field IMRT, indicating a superiority of IMRT over 3D-CRT in terms of normal tissue doses (26). In the current study, the superiority of SW-IMRT over FIF was further emphasised with better protection, especially for rectum and small intestine tissues.

Yang et al. performed a meta-analysis of 13 previous studies involving dosimetric comparison of 3D-CRT and IMRT for gynaecological malignancy. No significant differences were found between 3D-CRT and IMRT for the rectum at doses lower than $30 \mathrm{~Gy}$. By comparison, where the rectum had received $\geq 30 \mathrm{~Gy}$, doses were significantly reduced in IMRT compared with 3D-CRT. In the same study, the small intestine $\mathrm{V} 45$ values were lowered by $17.30 \%$ in IMRT. In addition, bone marrow and bladder irradiated volumes in IMRT were lower than in 3D-CRT (27). In this current study, similar results were obtained, with the mean rectum doses being considerably lower in SW-IMRT than for the FIF technique, especially at high doses. Because the rectum was adjacent to the PTV, SW-IMRT was able to protect the rectum better than the FIF technique. Moreover, the absolute volume of small intestine that received $\geq 45$ Gy was significantly decreased when compared with the other four FIF plans, which was found to be statistically significant $(\mathrm{p}<$ 0.001). Furthermore, the limited non-significant advantage for the bladder could be attributed to the overlapping of almost the whole bladder volume with PTV.

Duman et al. performed a dosimetric comparison of the different treatment planning techniques for gynaecological malignancies. Four different CI values, suggested by previous studies, and HI values derived from ICRU-83 were calculated for different plans, including 3D -CRT, FIF and IMRT. Mean results showed that the FIF technique was better than the other treatment techniques in terms of homogeneity, although no statistically significant difference

Int. J. Radiat. Res., Vol. 18 No. 4, October 2020 
was observed among others. When CI results were compared, FIF and IMRT with different number of fields showed superiority over 3D-CRT, and were statistically significant except for the SALT group expression (11). In this current study, the homogeneity of the FIF plans was found to be comparable with SW-IMRT, and even better in some cases. For the RTOG expression, a non-significant difference between the four FIF plans indicated that MLC margins were not an important variable for the HI value of a plan. However, this result should not lead one to a clinical inference. Although SW-IMRT was the least homogeneous plan with the RTOG expression, it was comparable with other FIF plans when evaluated based on the ICRU-83 report, which is accepted as the most illustrative and useful tool for $\mathrm{HI}$ in recent literature $(8,11,13,14)$. Furthermore, calculations based on ICRU suggestions showed that MLC margins could be the key parameter for the HI value in the FIF planning technique $(\mathrm{p}<0.001)$. On the other hand, SW-IMRT was significantly superior to all four FIF plans, giving a result closer to 1 and ensuring the best outcome in terms of conformity with the ICRU-62, RTOG 1993 and CIOPT suggestions $(\mathrm{p}<0.001)$.

Feuvret et al. reviewed previous studies related to different CI approaches (16). The review study deeply assessed the pros and cons of CI suggestions proposed by RTOG, the SALT group, Lomax and Scheib, van't Riet etal. and Baltas et al. $(14,18,19,20,28)$. The RTOG suggestion calculated the proportion of the target coverage from the corresponding isodose. Lefkopoulos et al., as the SALT group, introduced an approach that principally estimated the relation of RI volume with target volume. Lomax and Scheib introduced a CI expression that took normal tissue irradiation into consideration by calculating the RI volume outside the target volume. Baltas et al. utilised $\mathrm{CN}$ in brachytherapy by defining new parameters and critical organs. However, $\mathrm{CN}$, an expression suggested by van't Riet et al. could be accepted as the most well-turned expression (20). $\mathrm{CI}_{\text {OPT }}$ similarly explained both overlapping sections of RI, with target and excessive irradiation on adjacent healthy tissues. The supremacy of $\mathrm{CI}_{\mathrm{OPT}}$ over $\mathrm{CN}$ was due to a substantial expansion of the conventional range of the $\mathrm{CI}$ from $(-2)$ to $(+1)$ in order to highlight the potential differences among techniques. $\mathrm{CI}_{\text {OPT }}$ was more critical and precise on variations of $\mathrm{V}_{\mathrm{RI}}, \mathrm{V}_{\mathrm{PTV}}$, $V_{\text {OVERLAP, }} V_{\text {MISSING }}$ and $V_{\text {EXCESSIVE }}$ in Eqn.2, resulting in small variations of both the volumes of RI and PTV affecting the CI value significantly. The principle basis of the $\mathrm{CI}_{\text {opt }}$ formula was choosing the appropriate and preferred RI that would cover the target. The first and the most important step of the calculation process should be determining the specified RI. Although determination of the RI depends on clinician, considering RTOG protocol suggestions could also play a crucial role.

\section{CONCLUSION}

External radiation therapy is one of the most preferred treatment methods in gynecological cancers. Owing to the fact that 3D-CRT still being used in many radiation oncology departments, the FIF technique can be used to generate homogeneous treatment plans as for the SW-IMRT technique. In the case of normal tissue doses, MLC margins had a substantial influence on both $\mathrm{HI}$ and $\mathrm{CI}$. The new $\mathrm{CI}_{\mathrm{OPT}}$ formulation proposed in this study could be a useful plan evaluation tool in assessing the conformity of a treatment plan, being also compatible with the RTOG 1993 and ICRU-62 reports.

\section{Conflicts of interest: Declared none.}

\section{REFERENCES}

1. Creutzberg $\mathrm{CL}$, van Putten $\mathrm{WL}$, Koper PC, Lybeert $\mathrm{ML}$, Jobsen JJ, Wárlám-Rodenhuis CC, et al. (2000) Surgery and postoperative radiotherapy versus surgery alone for patients with stage-1 endometrial carcinoma: multicentre randomised trial. Lancet, 355: 1404-e1411.

2. Keys HM, Roberts JA, Brunetto VL, Zaino RJ, Spirtos NM, Bloss JD, et al. (2004) A phase III trial of surgery with or without adjunctive external pelvic radiation therapy in intermediate risk endometrial adenocarcinoma; a Gynecologic Oncology Group study. Gynecol Oncol, 92: 744-751.

3. Creutzberg $\mathrm{CL}$, Nout RA, Lybeert $\mathrm{ML}$, Wárlám?Rodenhuis CC, Jobsen JJ, Mens JW, et al. (2011) Fifteen?year radio- 


\section{Özseven and Özkan / Dosimetric evaluation of FIF and IMRT}

therapy outcomes of the randomized PORTEC? 1 trial for endometrial carcinoma. Int J Radiat Oncol Biol Phys, 81: e631⿴囗玉 638.

4. Creutzberg $\mathrm{CL}$, van Putten $\mathrm{WL}$, Koper PC, Lybeert $\mathrm{ML}$, Jobsen JJ, Wárlám? Rodenhuis CC, et al. (2001) The morbidity of treatment for patients with stage I endometrial cancer: results from a randomized trial. Int J Radiat Oncol Biol Phys, 51: 1246-1255.

5. Atiq M, Atiq A, Iqbal K, Shamsi $Q$, Andleeb F, Buzdar SA. (2017) Evaluation of dose conformity and coverage of target volume for intensity modulated radiotherapy of pelvic cancer treatment. Indian J Cancer, 54: 379-384.

6. Wagner A, Jhingran A, Gaffney D (2013) Intensity modulated radiotherapy in gynecologic cancers: hope, hype or hyperbole? Gynecol Oncol, 130: 229-236.

7. Elicin O, Atkovar G, Ergen ŞA, Ipek S, Karacam S, Şahinler I (2017) Dosimetric comparison of static field intensitymodulated radiotherapy and volumetric Modulated arc therapy for adjuvant treatment of patients with endometrial cancer. Turk J Oncol, 32(2): 70-76.

8. The International Commission on Radiation Units and Measurements (2010) Prescribing, recording, and reporting photon beam intensity modulated radiation therapy (IMRT). ICRU Report 83. J ICRU, 10: 1-106.

9. Small W Jr, Mell LK, Anderson P, Creutzberg C, De Los Santos J, Gaffney D, et al. (2008) Consensus guidelines for delineation of clinical target volume for intensity?modulated pelvic radiotherapy in postoperative treatment of endometrial and cervical cancer. Int I Radiat Oncol Biol Phys, 71: 428-434.

10. Guy JB, Falk AT, Auberdiac P, Cartier L, Vallard A, Ollier E, et al. (2016) Dosimetric study of volumetric arc modulation with RapidArc and intensity-modulated radiotherapy in patients with cervical cancer and comparison with 3dimensional conformal technique for definitive radiotherapy in patients with cervical cancer. Medical Dosimetry, 41: 9-14.

11. Duman E, Inal A, Sengul A, Koca T, Cecen Y, Yavuz MN (2016) Dosimetric comparison of different treatment planning techniques with International Commission on Radiation Units and Measurements Report-83 recommendations in adjuvant pelvic radiotherapy of gynecological malignancies. J Can Res Ther, 12: 975-980.

12. Akpati H, Kim C, Kim B, Park T, Meek A (2008) Unified dosimetry index (UDI): a figure of merit for ranking treatment plans. J Appl Clin Med Phys, 9(3): 99-108.

13. Krishnan J, Shetty J, Rao S, Hegde S, Shambhavi C (2017) Comparison of Rapid Arc and intensity-modulated radiotherapy plans using unified Dosimetry Index and the Impact of Conformity Index on unified dosimetry index evaluation. J Med Phys, 42(1): 14-17.

14. Shaw E, Kline R, Gillin M, Souhami L, Hirschfeld A, Dinapoli $\mathrm{R}$, et al. (1993) Radiation Therapy Oncology Group: radiosurgery quality assurance guidelines. Int I Radiat Oncol Biol Phys, 27: 1231-1239.

15. International Commission on Radiation Units and Measurements (1999) Prescribing, recording and reporting photon beam therapy (Supplement to ICRU Report 50). ICRU Report 62. ICRU. Bethesda, USA.

16. Feuvret L, Noël G, Mazeron JJ, Bey P (2006) Conformity index: a review. Int J Radiat Oncol Biol Phys, 64(2): 333342.
17. Knöös T, Kristensen I, Nilsson P (1998) Volumetric and dosimetric evaluation of radiation treatment plans: radiation conformity index. Int I Radiat Oncol Biol Phys, 42: $1169-1176$.

18. Lefkopoulos D, Dejean C, El-Balaa H, Platoni K, Grandjean $P$, Foulquier J, et al. (2000) Determination of dose-volumes parameters to characterise the conformity of stereotactic treatment plans. In: The Use of Computers in Radiation Therapy, (Schlegel W and Bortfeld T, eds.), Springer, Berlin, Germany.

19. Lomax NJ and Scheib SG (2003) Quantifying the degree of conformity in radiosurgery treatment planning. Int J Radiat Oncol Biol Phys, 55: 1409-1419.

20. van't Riet $A$, Mak AC, Moerland MA, Elders LH, van der Zee W (1997) A conformation number to quantify the degree of conformality in brachytherapy and external beam irradiation: application to the prostate. Int J Radiat Oncol Biol Phys, 37(3): 731-736.

21. RTOG 0418: A phase II study of intensity modulated radiation therapy (IMRT) to the pelvis +/ chemotherapy for postoperative patients with either endometrial or cervical carcinoma: Current Version Date: 8/17/ 2011 Available from: https://www.rtog.org/ClinicalTrials/ProtocolTable/ Study Details.aspx?study $=0418$

22. RTOG 1203: A randomized phase III study of standard vs. IMRT pelvic radiation for post-operative treatment of endometrial and cervical cancer (TIME-C)-RTOG CCOP Study: Current Version Date: 3/16/ 2015. Available from: https://www.rtog.org/ClinicalTrials/ProtocolTable/ StudyDetails. aspx?action $=$ openFile\&FileID $=9644$

23. Marks LB, Yorke ED, Jackson A, Ten Haken RK, Constine LS, Eisbruch A, et al. (2010) Use of normal tissue complication probability models in the clinic. Int I Radiat Oncol Biol Phys, 76, (3); Supplement, pp. S10-S19.

24. Kavanagh BD, Pan CC, Dawson LA, Das SK, Li XA, Ten Haken RK, et al. (2010) Radiation dose-volume effects in the stomach and small bowel. Int J Radiat Oncol Biol Phys, 76, (3); Supplement, pp. S101-S107.

25. Yavas G, Yavas C, Acar H, Buyukyoruk A, Cobanoglu G, Kerimoglu OS, et al. (2013) Dosimetric comparison of 3dimensional conformal and field-in-field radiotherapy techniques for the adjuvant treatment of early stage endometrial cancer. Phys Med, 29(6): 577-582.

26. Heron DE, Gerszten K, Selvaraj RN, King GC, Sonnik D, Gallion $\mathrm{H}$, et al. (2003) Conventional 3D conformal versus intensity-modulated radiotherapy for the adjuvant treatment of gynecologic malignancies: a comparative dosimetric study of dose-volume histograms. Gynecol Oncol, 91: 39-45.

27. Yang B, Zhu L, Cheng H, Li Q, Zhang Y, Zhao Y (2012) Dosimetric comparison of intensity modulated radiotherapy and three?dimensional conformal radiotherapy in patients with gynecologic malignancies: a systematic review and meta? ?analysis. Radiat Oncol, 7:197.

28. Baltas D, Kolotas C, Geramani K, Mould RF, loannidis G, Kekchidi M, et al. (1998) A conformal index (COIN) to evaluate implant quality and dose specification in brachytherapy. Int J Radiat Oncol Biol Phys, 40: 515-524.

Int. J. Radiat. Res., Vol. 18 No. 4, October 2020 